\title{
ELECTROMAGNETIC INTERFERENCE (EMI) PRODUCED BY HIGH VOLTAGE TRANSMISSION LINES
}

\author{
Ban M. Alameri \\ Department of Electrical Engineering \\ Al-Mustansiriya University \\ P.O. Box 14132, Baghdad, Iraq \\ Ban.alameri@uomstansiriyah.edu.iq
}

\begin{abstract}
Electromagnetic interference in high voltage transmission lines has been an interest topic due to its effect on human health, plants, electrical and telecommunication equipment. Extremely high voltages (EHV) in transmission lines are reasons of electrostatic effects, while short circuit currents and line loading currents are responsible for electromagnetic effects.

The aim of this research is to analyze electromagnetic fields in high voltage transmission lines in theoretical study and calculating its level in overhead T. L and therefore estimated the EMI produced, by employing a mathematical model of $230 \mathrm{KV}$ tower double circuit configurations of high voltage transmission lines. The calculation is based on computer aided analysis (CAA) by using fields and corona effects software (FACE). It's found that the overhead power lines of general frequency $(50 \mathrm{~Hz})$ generates a highly intense magnetic field, the electromagnetic fields depends on the distance from sources and the type of line configuration. They decrease as the distance increase from the tower and conductors and increase with a high current.

The strength of an electric field is proportional to the voltage of the line and the magnetic field strength is proportional to the current in the high voltage transmission lines. Distribution line with a high current load may produce a magnetic field that is as high as those produced by some high voltage transmission lines.

Some techniques of reduction of the effects of electromagnetic interference have suggested such as rearrangement conductors of transmission line, and distance from phase conductor and grounding system. The study recommended to keep safety distance operation in high voltage transmission lines with the necessity for engineer to take into account the effect of electromagnetic interference in the design stage of high voltage transmission power system, and to avoid any addition cost may be occur due to neglected effects of electromagnetic interference that produces by high voltage transmission lines.
\end{abstract}

Keywords: electric field, electromagnetic interference (EMI), high voltage transmission lines (HVTL), field effects.

DOI: $10.21303 / 2461-4262.2020 .001398$

\section{Introduction}

Nowadays, the increasing of power demand has increased the need for transmitting a bulk of power over long distance.Electric and magnetic fields, referred to electromagnetic fields (EMF) as shown in Fig. 1 the magnetic field strength lines of along conductor with an electric current passing through it, have a circular shape.

EMF occurs as a result of the power generation, transmission and distribution of electric power. High voltage transmission lines with high currents levels produce large values of electric and magnetic field stresses which produce an electromagnetic interference [1].

Electromagnetic interference has impact on health human, plants and the nearby objects such as electrical substation, buried pipelines, houses and telecommunication equipment mainly operating in frequency range below UHF [2].

Electromagnetic interferences is evaluated based on the knowledge of the current or voltage of lines by calculating the electrical and magnetic fields around the conductor of transmission lines [2].

The most important factors it is necessary to take into account for evaluation the electromagnetic fields are:

- frequency;

- distance;

- current;

- loading on power lines;

- and height of tower.

The block diagram of electromagnetic fields and its effects are shown in Fig. 2. 


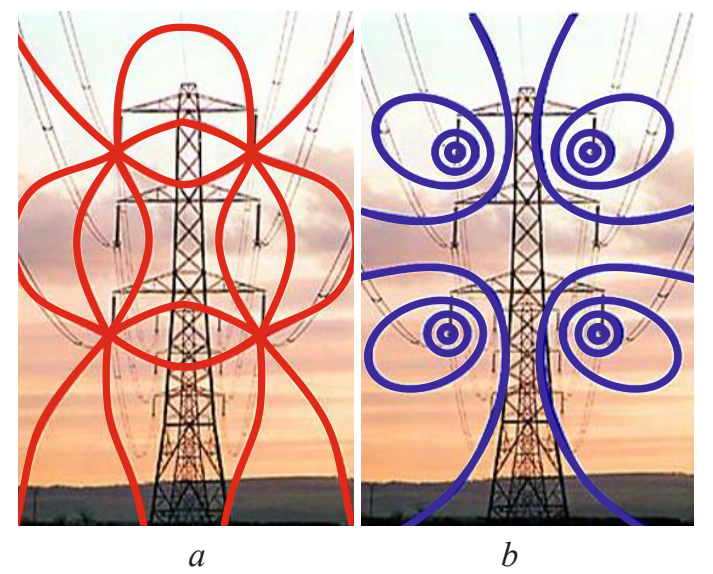

Fig. 1. The electromagnetic fields in high voltage T.L: $a$ - Magnetic field; $b$ - electric field

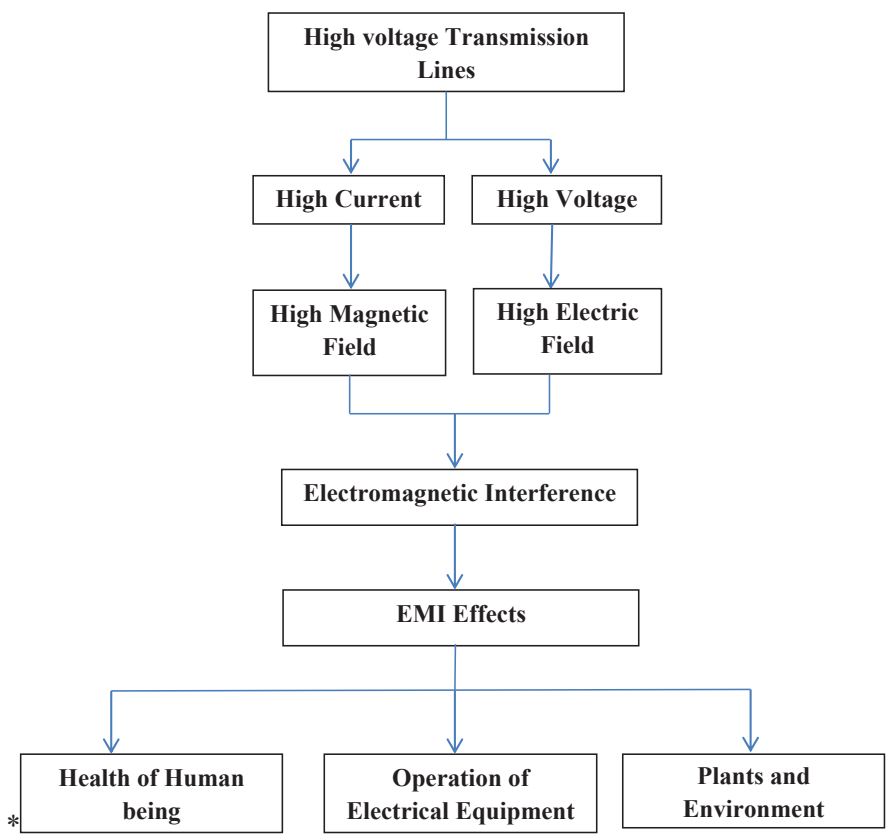

Fig. 2. Block diagram of electromagnetic interferences in high voltage and its effects

The main domains which may be affected by electromagnetic interferences which generated from electromagnetic fields of overhead lines are:

a) health of human being: electromagnetic field EMF can cause a danger to people, and long-term effects which produce health problems and danger of electric shock from high electromagnetic fields, the sanitary permissible standards are violated and measures must be taken (the norms of EMF standard 2004), the effective values of magnetic field strength should not exceed $40 \mu \mathrm{T}$ in the residential environments [3].

b) operation of electrical equipment: electromagnetic fields have effect on the electrical protective equipment's, especially in the high voltage substations. The electromagnetic interferences of high voltage transmission lines cause electrical currents inside any substation in range of the field, and induced voltage in the pipeline [4]. Plants and environment: Recent studies of electromagnetic fields (EMF) effects on the environmental have focused on extremely low frequency (ELF), as those generated by overhead power lines. They explained that plants are the most sensitive to EMF, and effect on alanine (in plants) and proteins (in animals) that change the growth of certain plant cells [5]. 


\section{Field Analysis due to overhead lines}

The only way to be the safest from the electromagnetic interferences exposure generated in high voltage transmission lines is to calculate, or predict the electric and magnetic field values using theoretical or experimental techniques [6].

\section{1. Electric Field Strength}

In the ground level, the electric field strength can be calculated due to potential of the conductor at a height $(H)$, and phase conductors are considered as infinite line charges. The charge $(Q)$ on the conductor is given by:

$$
Q=C \cdot V
$$

where $C$ is the capacitance of the conductor with respect to the earth in coulombs/m; $V$ is the voltage in volt unit.

If there are several conductors with capacitance $(C i j),(V i j)$ is the voltage of the $j$-th conductor to earth. The sum of charges is given by:

$$
Q=\sum C i j \cdot V j
$$

The contribution from the charge $Q i$ on conductor $i$ from charge - Qi on the image of this conductor as shown in Fig. 3.

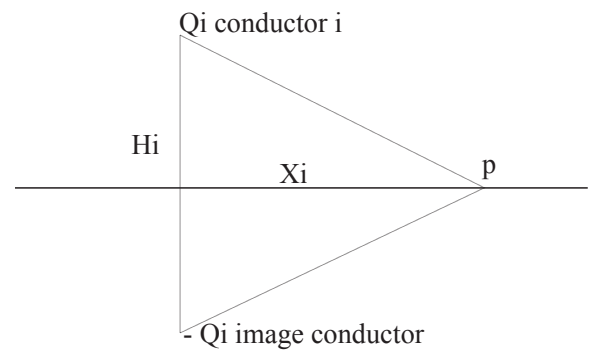

Fig. 3. Field strength at point $\mathrm{P}$ due to conductor i

The electric field around the conductor of transmission line can be calculated by using image theory at point $(P)$. Then, the field strength $(E i)$ in units of $\mathrm{KV} / \mathrm{m}$ is given by:

$$
E i=\frac{Q i}{2 \pi * \varepsilon_{0}} \frac{H i}{H i^{2}+X i^{2}},
$$

where $\mathrm{Hi}$ is the vertical distance to the point of measurements; $\mathrm{Xi}$ is the Horizontal distance to the point of measurements; $\varepsilon_{0}$ is the relative Permittivity of air.

The total electric field strength at point $P$ is the sum of all electric fields due to various conductors [7].

\section{2. Magnetic Field Strength}

The magnetic field is created by the current carrying in overhead conductor and each point of charge will produce a magnetic field in $x$-direction and $y$-direction [8]. The magnetic field of vertical and horizontal component is given by:

$$
\begin{aligned}
& B v=\sum_{n=1}^{n}\left(-2 x 10^{-3}\right) \cdot I_{n}\left[\frac{y-y_{n}}{m^{2}} \cdot \frac{y-y_{n}+a}{m i^{2}}\right], \\
& B h=\sum_{n=1}^{n}\left(-2 x 10^{-3}\right) \cdot I_{n}\left[\frac{x-x_{n}}{m^{2}} \cdot \frac{x-x_{n}+a}{m i^{2}}\right],
\end{aligned}
$$


where In represents the current for $n$-th conductor in ampere unit and can be evaluated by:

$$
\begin{gathered}
\text { In }=\frac{P}{\sqrt{3} U \cos (\varphi)}, \\
a=\sqrt{\frac{1}{\pi \mu \sigma f}},
\end{gathered}
$$

$m$ is distance from point $p$ to conductor $n ; m i$ is distance from point $p$ to the image conductor $n ; x$, $y$ are coordinates of point $p ; y_{n}, x_{n}$ are coordinates of conductor $n . ; f$ - frequency.

The magnetic field strength is:

$$
B=\sqrt{B v^{2}+B h^{2}},
$$

$B v$ is magnetic field strength vectors in the vertical direction in Gauss; $B h$ is magnetic field strength vectors in the horizontal direction in Gauss;

\section{Mathematical Model}

A digital modeling is selected as a method for investigation the electromagnetic fields produced by the $230 \mathrm{KV}$ overhead transmission lines. The electric field is proportional to voltage, so it will be constant with constant voltage, and the magnetic field is proportional to current which varies greatly as the power demanded on the transmission line varies.

Calculation of electromagnetic field around HVTL will be in different level depending on the height above the ground and the distance from transmission line.

The preferred height above the earth is one meter, and it's specified in the ANSI/IEEE standard [9].

In order to know the value of electromagnetic fields under the transmission line, one meter height above the earth with various distances has been performed for horizontal and vertical tower configuration and for double circuit as in Fig. 4. The calculation is based on computer aided analysis (CAA) by fields and corona effects software (FACE).

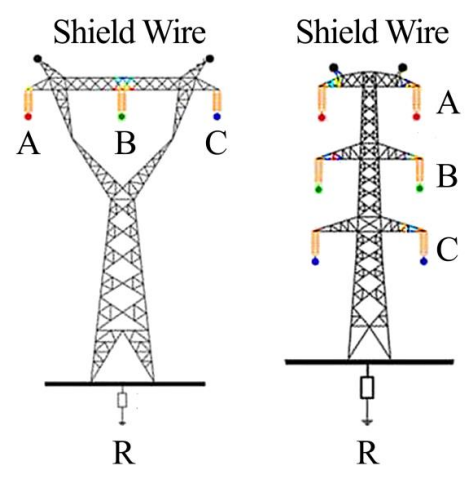

Fig. 4. Horizontal and Vertical Tower Configuration

Electromagnetic fields calculation requires modeling of transmission line and basic electromagnetic theory application. In this study, a mathematical model has considered a high voltage power line of $230 \mathrm{KV}$ tower of double circuit configuration Fig. 5, considers a mathematical model of HVTL with characteristics as follows.

The $230 \mathrm{KV}$ double circuit power T.L, 3-phase line, the phase current $I n=500$ ampere, for obtaining a result of the mathematical calculation accurate as possible, 11 total position of point $\mathrm{P}$ were selected, spaced by 3 meter and located at 1 meter vertically from the earth level. The diameter of each phase conductor is $0.4 \mathrm{~mm}$. The sag of the double circuit $230 \mathrm{KV}$ transmission lines is not taken into consideration. 


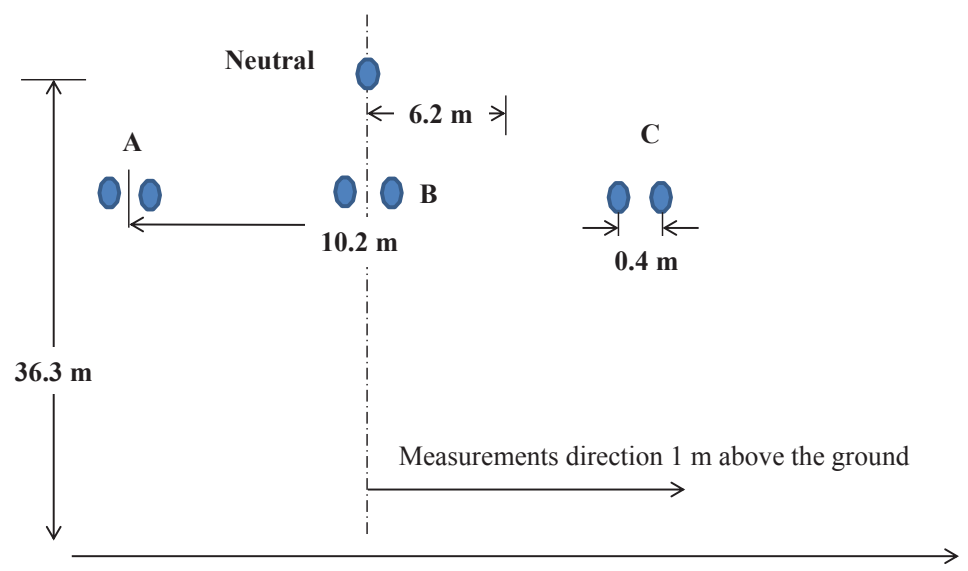

Fig. 5. Tower configuration of $230 \mathrm{KV}$

The data for the three phase conductors and corresponding position in the tower, the input parameters for fields' strength calculations are as follows in Table 1.

Table 1

Parameters data for fields' calculations

\begin{tabular}{cccccc}
\hline Phase & Position x-axis (m) & Position y-axis (m) & Voltage (r.m.s) KV & Phase angle (Degree) & Current KA \\
\hline Phase A & 0 & 31.3 & 132.8 & 0 & 0.5 \\
Phase B & 10.2 & 26.3 & 132.8 & 120 & 0.5 \\
Phase C & 20.4 & 22 & 132.8 & 240 & 0.5 \\
Neutral & 16.4 & 36.3 & 0.8 & 0 & 0
\end{tabular}

\section{Results}

The results obtained from mathematical presented calculation of electric and magnetic fields level for 3-phase conductor and corresponding of vertical and horizontal position in the tower with the respect of model in Fig. 5, and less than one meter of the high voltage transmission line is as follows.

The calculations results in Table 2 and curve in Fig. $\mathbf{6}$ shows the electrical field strength for vertical and horizontal configurations with varying distance. As the distance increases from the tower and the conductors, the electrical field strength decreases rapidly and achieves its maximum value at point (P3) is $1.022 \mathrm{KV} / \mathrm{m}$ as shown in Fig. 7.

Table 2

The calculations of the electric field strength

\begin{tabular}{cccccc}
\hline Point & Distance $(\mathbf{m})$ & E-Field KV/m & Theta (Degree) & Ex-Field KV/m & Ey-Field KV/m \\
\hline P1 & 0 & 0.755 & 87.20 & 0.193 & 0.754 \\
P2 & 3 & 0.889 & 84.60 & 0.195 & 0.886 \\
P3 & 6 & 1.022 & 88.00 & 0.135 & 1.021 \\
P4 & 9 & 1.002 & 92.70 & 0.107 & 1.001 \\
P5 & 12 & 0.850 & 96.10 & 0.118 & 0.845 \\
P6 & 15 & 0.661 & 97.70 & 0.107 & 0.655 \\
P7 & 18 & 0.499 & 98.00 & 0.083 & 0.494 \\
P8 & 21 & 0.379 & 97.60 & 0.060 & 0.376 \\
P9 & 24 & 0.294 & 96.90 & 0.042 & 0.292 \\
P10 & 27 & 0.234 & 96.30 & 0.030 & 0.232 \\
P11 & 30 & 0.188 & 95.60 & 0.019 & 0.162
\end{tabular}




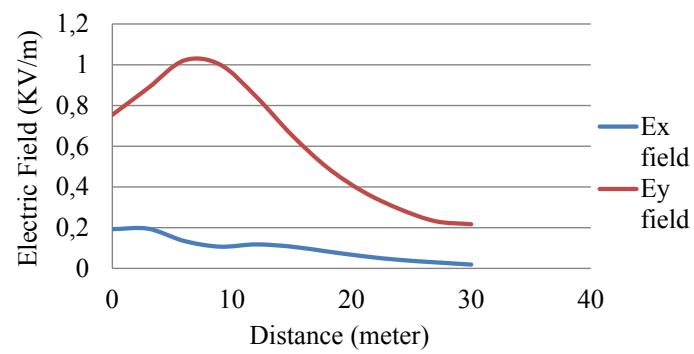

Fig. 6. Electric field strength for horizontal and vertical configuration

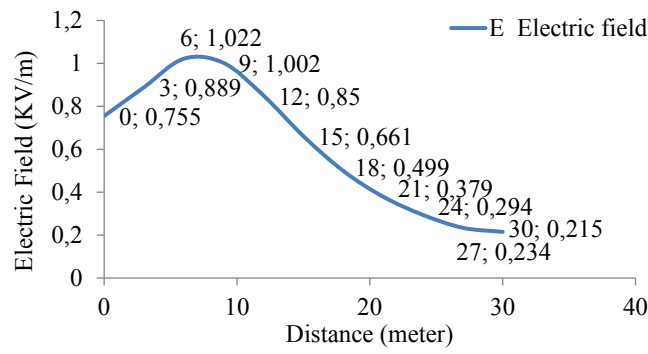

Fig. 7. Overall electric field strength in $230 \mathrm{KV}$ HVTL varying with distance

The calculations obtained in Table 3, and Fig. 8 shows the magnetic field strength curves for vertical and horizontal configuration with varying distance. It can be seen that the magnetic field strength value decreases in horizontal direction from the tower. The maximum magnetic field level observed in this $230 \mathrm{KV}$ tower at point (P2) is 0.03 Gauss as shown in Fig. 9.

Table 3

The calculations of the magnetic field strength

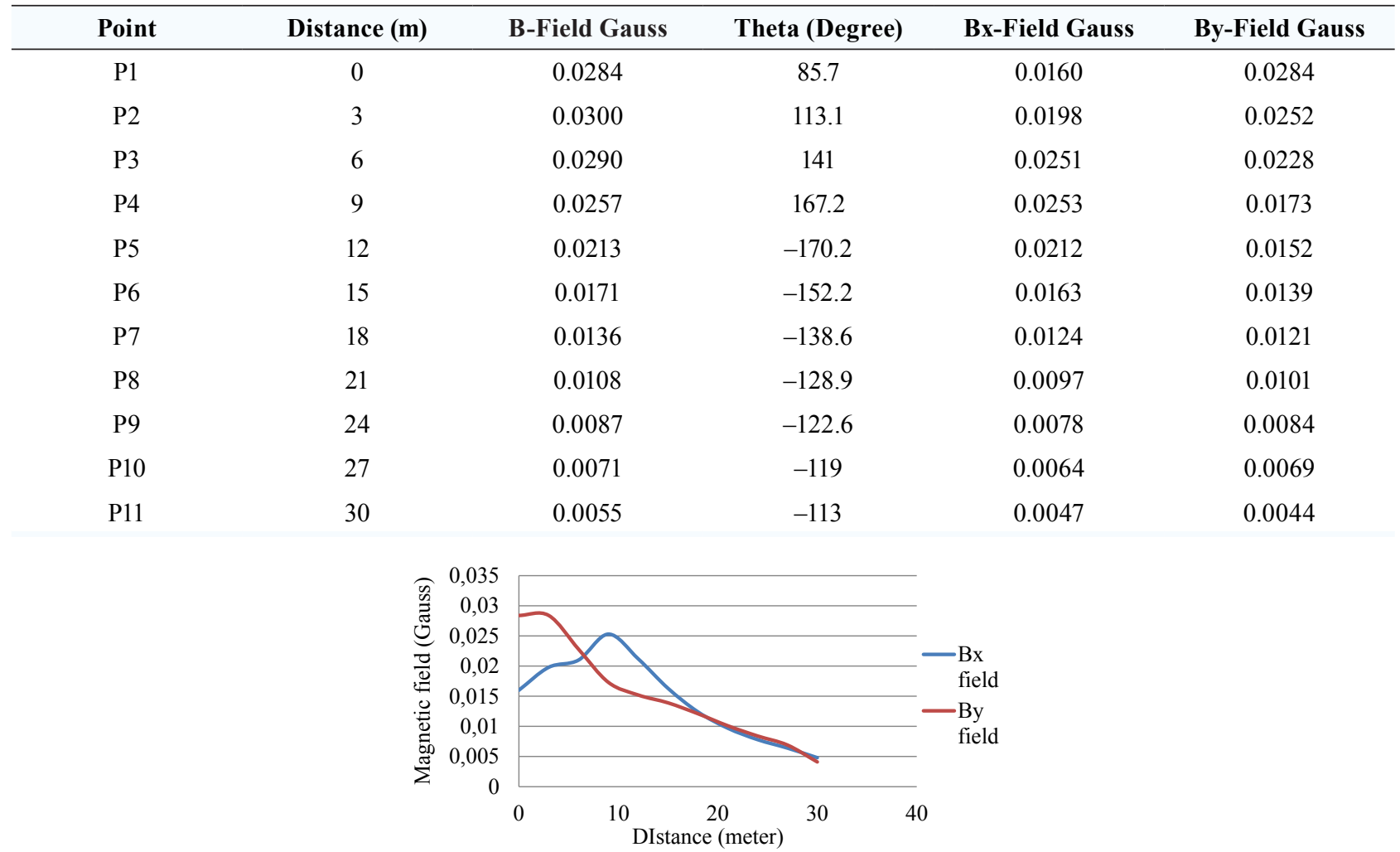

Fig. 8. Magnetic field strength for horizontal and vertical configuration 


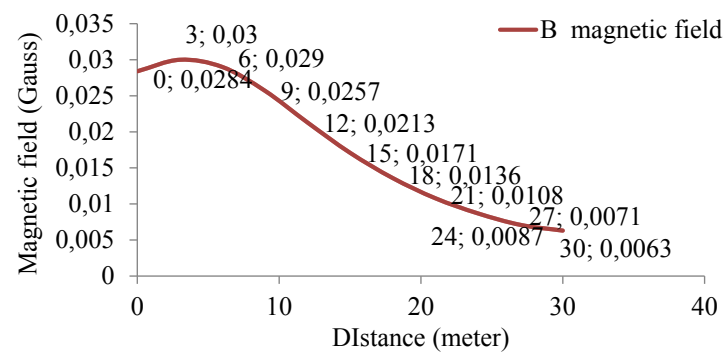

Fig. 9. Overall magnetic Field strength in 230 KV HVTL varying with distance

From the result above, let's observe that the magnitude of electromagnetic field in the vertical configuration, especially for magnetic field, is higher than its horizontal configuration.

At constant voltage, the electric field will be constant. With increasing the current carrying will not change the electric field, but will increase the magnetic field. Consequently, it can be estimated that the levels of magnetic field are effected to produce an interference more than electric field.

For reducing the effect of the electromagnetic interference produced by high voltage transmission lines, let's focus on the approaches available for the reduction of magnetic field near overhead lines on next section.

\section{Reduction approaches of electromagnetic interference}

There are some approaches for reduction of electromagnetic field near the high voltage transmission lines. The using of suitable approaches depends on its advantage, disadvantages and cost [10].

Reducing the magnetic field as a function of phase current, rearrangement conductors of transmission line, distance from phase conductor and grounding system.

Rearrangement of transmission line conductors can reduce electromagnetic interference of HVTL. It's an efficient method, but it is important to estimate the cost of using this solution.

The larger distance of the phase conductor from the ground, will produce smaller magnetic field level. The conductor clearance is related to the system voltage and cost of high voltage tower.

To overcome the effect of the electromagnetic interference radiation from high voltage transmission lines can be fixed by grounding all electrical equipments.

\section{Discussion of mathematical results}

The results of study explains, the calculations of electromagnetic fields in $230 \mathrm{KV}$ high voltage lines in horizontal and vertical configuration, with varying distance from the tower, a maximum level of electromagnetic field has produced a high interference in the same distance.

This study proposed a mathematical model of $230 \mathrm{KV}$ tower of double circuit configuration which it's not study in previous researches, the calculation of simulation model made by field and corona software (FACE), and the measurements performed in closed distance from TL varying (0-30) meter.

Setting standards of maximum level of EMF radiation in transmission lines is difficult to determine the long term effects which cause health problems and damage for some electrical equipments such as substation, also the theoretical calculation is not accurate like the practical measurements, its exist an error ratio in the measuring.

For further researches and development the study can be taken into account more parameters of transmission lines for evaluated the level of electromagnetic fields like height of tower, type of conductor and weather condition.

\section{Conclusions}

This study was focused on analysis and evaluation of electromagnetic fields in high voltage transmission lines, which caused an interferences and effects on other equipments. It is based on the calculations of electric and magnetic fields strength performed by fields and corona effects 
software (FACE) on $230 \mathrm{KV}$ tower model with various distances from the conductors in the vertical and horizontal configuration. By comparing the results of calculation, it is possible to observe that the electromagnetic field decreases with the increased distance from the conductor in the horizontal direction away from the tower. Some techniques for reduction of the effect of electromagnetic interference have been discussed.The study recommends for taking in account the effect of EMI in the design stage of the HVTL to avoid the problem of interference which requires huge expenses for handling it.

It is recommended that practical measurements of electromagnetic fields could be taken in locations, which depends on different weather conditions.

\section{References}

[1] Sinik, V., Despotovic, Z., Radovanovic, L. (2013). Influence of Non-Ionizing Radiation on the Environment. Conference: International Science Conference REPORTING FOR SUSTAINABILITY, 465-469.

[2] Silva Ortega, J., Cardenas Escorcia, Y., Valencia-Ochoa, G. (2018). Monitoring Electromagnetic Fields and Safe Operation Levels in Electrical Power Transmission Lines. Chemical Engineering Transactions, 67, 715-720. doi: https://doi.org/10.3303/ CET1867120

[3] Raghu, N., Nagendra, K. (2017). Survey of Electromagnetic Field Near EHV Lines and Their Health Hazards. Conference: ICspace-2017, 372-377.

[4] Ali, Y. S. M., El-Baset, A. A., Elghaffar, A. N. A. (2016). Mathematical calculation of Electromagnetic field in high voltage substation to treatment its effect on the protective equipments. International journal of engineering, XIV.

[5] Dervic, K., Jankovic, S., Despotovic, Z. V., Sinik, V. (2012). The radiation of electromagnetic fields of very low frequency. Conference: II International Conference ECOLOGY OF URBAN AREAS 2012. Vol. 1. Zrenjanin, 348-356.

[6] Effects of High Voltage Transmission Lines on Humans and Plants. Available at: https:/electricalnotes.wordpress. com/2012/02/17/effects-of-high-voltage-transmission-lines-on-humans-and-plants/

[7] Ouadah, M., Zergoug, M. (2014). Analysis of the Electromagnetic Interferences between Overhead Power Lines and Buried Pipelines. Mediterranean Journal of Modeling and Simulation, MJMS, 01, 013-023.

[8] Natarajan, R. (2002). Computer-Aided Power System Analysis. Boca Raton: CRC Press, 392. doi: https://doi.org/10.1201/ 9780203910832

[9] Height above ground for measurement (2017). Energy Network Association.

[10] Rycroft, M. (2018). The Mitigation of electric and magnetic fields from transmission lines. EE Publishers. Technology and Business for development. 